OPEN ACCESS

Edited by:

Andy Pereira

University of Arkansas, United States

Reviewed by:

Longxing $\mathrm{Hu}$,

Hunan Agricultural University, China

Omar Borsani,

University of the Republic, Uruguay

*Correspondence:

Jie Sun

jetsun@mail.scuec.edu.cn

Jinmin Fu

jinminfu@gmail.com

Received: 10 May 2017

Accepted: 24 July 2017

Published: 08 August 2017

Citation:

Chen $K$, Zhang $M$, Zhu $H$, Huang $M$,

Zhu Q, Tang D, Han X, Li J, Sun J and Fu J (2017) Ascorbic Acid Alleviates Damage from Heat Stress in the Photosystem II of Tall Fescue in Both the Photochemical and Thermal Phases.

Front. Plant Sci. 8:1373. doi: 10.3389/fp/s.2017.01373

\section{Ascorbic Acid Alleviates Damage from Heat Stress in the Photosystem II of Tall Fescue in Both the Photochemical and Thermal Phases}

\author{
Ke Chen 1,2,3, Minna Zhang ${ }^{1}$, Huihui Zhu' ${ }^{1}$, Meiyu Huang ${ }^{1}$, Qing Zhu ${ }^{4}$, Diyong Tang ${ }^{1}$, \\ Xiaole Han ${ }^{1,3}$, Jinlin $\mathrm{Li}^{3}$, Jie Sun ${ }^{1,3 *}$ and Jinmin $\mathrm{Fu}^{2 *}$ \\ ${ }^{1}$ College of Resources and Environmental Science, South-Central University for Nationalities, Wuhan, China, ${ }^{2}$ Key \\ Laboratory of Plant Germplasm Enhancement and Specialty Agriculture, Wuhan Botanical Garden, The Chinese Academy of \\ Sciences, Wuhan, China, ${ }^{3}$ Key Laboratory of Catalysis and Materials Science of the State Ethnic Affairs Commission \& \\ Ministry of Education, College of Resources and Environmental Science, South-Central University for Nationalities, Wuhan, \\ China, ${ }^{4}$ Wuhan Kaidi Electric Power Environmental Co., Ltd., Wuhan, China
}

L-Ascorbate (Asc) plays important roles in plant development, hormone signaling, the cell cycle and cellular redox system, etc. The higher content of Asc in plant chloroplasts indicates its important role in the photosystem. The objective of this study was to study the roles of Asc in tall fescue leaves against heat stress. After a heat stress treatment, we observed a lower value of the maximum quantum yield for primary photochemistry $(\varphi \mathrm{Po})$, which reflects the inhibited activity of the photochemical phase of photosystem II (PSII). Moreover, we observed a higher value of efficiency of electron transfer from $Q_{B}$ to photosystem I acceptors $\left(\delta_{R O}\right)$, which reflects elevated activity of the thermal phase of the photosystem of the tall fescue. The addition of Asc facilitate the behavior of the photochemical phase of the PSIl by lowering the ROS content as well as that of the alternative electron donor to provide electron to the tyrosine residue of the D1 protein. Additionally, exogenous Asc reduces the activity of the thermal phase of the photosystem, which could contribute to the limitation of energy input into the photosystem in tall fescue against heat stress. Synthesis of the Asc increased under heat stress treatment. However, under heat stress this regulation does not occur at the transcription level and requires further study.

Keywords: L-ascorbate, heat stress, tall fescue, photochemical phase, thermal phase

\section{INTRODUCTION}

Grasslands occupy about twice the land area of grain crops throughout the world (Mian et al., 2008) and play important ecological roles in the biosphere. For example, grasslands influence the carbon cycle, as well as the nitrogen cycle. Tall fescue (Festuca arundinacea Schreb) grows in temperate regions of the world, which is a major cool season forage and turf grass species (Chen et al., 2013; Wang and Huang, 2017). Additionally, it has been widely reported to be a model species for the rhizoremediation of various organic contaminants because of its well-developed root system and broad adaptability to different climates and environments ( $\mathrm{Lu}$ and Zhang, 2014). Although tall fescue has a relatively higher thermal tolerance than other cool season turfgrasses, heat stress is still a major abiotic stress that limits its use across the world. Heat stress limits the growth and 
development of tall fescue in transitional and warm climatic regions, especially in summer, when high temperature can constrain growth, reduce turf quality, induce leaf withering and inhibit photosynthesis (Sun et al., 2015).

The photosynthetic activity of the chloroplasts is considered as the most heat sensitive cell function (Essemine et al., 2011). The linear electron transport chain in thylakoid membrane starts with the splitting of water and ends with the reduction of $\mathrm{NADP}^{+}$. Chlorophyll $a$ fluorescence, which is measured under saturating continuous light, is a highly sensitive, nondestructive, and reliable tool for studying the energy flow and photosynthetic efficiency in the photosystem (Stirbet and Govindjee, 2011). OJIP curves from chlorophyll $a$ fluorescence studies can be generally divided to photochemical phase and thermal phase. The photochemical phase is mainly related to the reduction of quinone $A\left(Q_{A}\right)$, and the thermal phase follows the photochemical phase, which disappears at subfreezing temperatures (Stirbet, 2012). In particular, Photosystem II (PSII) oxidizes water through an oxygen evolution complex (OEC, namely, the water splitting complex), which contains five oxygen atoms in addition to four $\mathrm{Mn}$ atoms and one $\mathrm{Ca}$ atom, which form a $\mathrm{Mn}_{4} \mathrm{CaO}_{5}$-cluster (Umena et al., 2011) and produce high amount of reactive oxygen species (ROS) in plants (Pfannschmidt, 2003; Pospíšil, 2012). The ROS are responsible for heat-induced damage to PSII (Yamashita et al., 2008). The D1 protein in the reaction center (RC) in PSII accepts the electron from the splitting of water by OEC and transfers it to plastoquinone molecules (PQ) through photochemical reactions, and the heat stress induces the cleavage of the DE-loop of the D1 protein.

L-Ascorbate (Asc) has crucial roles in plants, although its major plant synthesis pathway, the L-galactose pathway, has only been confirmed in the past decade (Smirnoff and Wheeler, 2000; Linster and Clarke, 2008; Smirnoff, 2011). It is one of the most abundant low molecular weight antioxidants in plants, which also play important roles in plant development, hormone signaling, the cell cycle and expansion, the cellular redox system and cofactors for enzymes (Debolt et al., 2007; Foyer and Noctor, 2011; Mellidou et al., 2012). In leaves, the concentration of Asc is approximately 1-5 mM, whereas the content of Asc in chloroplast is approximately $25 \mathrm{mM}$, which suggests that it may play an important role in photosynthesis and transmembrane electron transport (Wheeler et al., 1998). Under heat stress, the OEC is damaged due to the removal of extrinsic proteins and the release of calcium and manganese ions from their binding sites (Barra et al., 2005), which results in the blocking of electron transport from OEC to the $\mathrm{Try}_{z}{ }^{+}$(D1 protein, Tyr161). Moreover, it has been showed that under heat stress, Asc, as a large pool of alternative electron donors, could donate electrons to PSII to sustain electron transport activities in leaves (Toth et al., 2009, 2011).

In this study, we aimed to determine whether Asc could alleviate heat stress induced damage to tall fescue PSII. To this purpose, exogenous addition of Asc was conducted to study the role of Asc in tall fescue leaves against heat stress. Chlorophyll a fluorescence measurements were employed to study both the effects of Asc on the photosynthetic apparatus as well as the productivity of photosynthetic organisms. Additionally, thylakoid membranes of the tall fescue leaves were extracted to verify the ability of the Asc to serve as an alternative electron donor. Additionally, regulation of Asc synthesis under heat stress was studied and discussed to increase our understanding of the role of Asc against abiotic stress.

\section{MATERIALS AND METHODS}

\section{Plant Materials and Growth Conditions}

Tall fescue commercial type 'Huntdog 5' was seeded in pots with sand as growth medium (Luo et al., 2011). After germination, plants were kept in a greenhouse (day/night temperature: $24 / 20^{\circ} \mathrm{C}$ ) with a $16 \mathrm{~h}$ photoperiod (light intensity: $300 \mu \mathrm{mol}$ photons $\mathrm{m}^{-2} \mathrm{~s}^{-1}$ ) for 50 days to allow the tall fescue roots and shoots to be established (Fu and Huang, 2001). The seedlings were fertilized twice a week with $50 \mathrm{~mL}$ of half-strength Hoagland's solution.

\section{Reagent and Heat Stress Treatment}

Reagent treatments for the leaves were performed by a vacuum infiltration method since it supports high efficiency transport of reagents into plant leaves (Filippou et al., 2012). In this study, $20 \mathrm{mM}$ sodium ascorbate (Sigma, St. Louis, MO, United States) was used for exogenous addition of ascorbic acid (marked as "Asc" in the figures and tables) (Uchida et al., 2002). Briefly, the third fully expanded leaves were cut from a petiole and immersed in $1 / 2$ strength Hoagland's solution alone (marked as " $\mathrm{Ct}$ " in the figures and tables) or with Asc in a $15-\mathrm{cm}$ petri dish. Then, the petri dishes were moved into a desiccator for vacuum infiltration for $15 \mathrm{~min}$ in the dark (Ederli et al., 2006; Attallah et al., 2007).

Heat stress treatments of tall fescue leaves were conducted using the same methods as our previous research (Chen et al., 2013). Briefly, after the reagent treatments, the petioles of the leaves were immersed in $1 \mathrm{~cm}$ of $1 / 2$ strength Hoagland's solution and maintained in a falcon tube with Asc or no Asc. Then, the tubes were placed in a growth cabinet with a temperature of 24

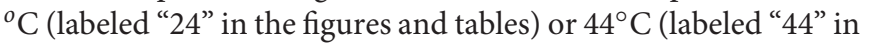
the figures and tables), and a light intensity of $100 \mu \mathrm{mol}$ photons $\mathrm{m}^{-2} \mathrm{~s}^{-1}$, and humidity of $80 \%$ for the indicated durations. After the treatments, the leaves were subjected to variety analysis.

\section{Chlorophyll a Fluorescence Transient}

In our study, all measurements related to $\mathrm{Chl} a$ fluorescence were measured by a PAM chlorophyll fluorometer (PAM 2500, Heinz Walz $\mathrm{GmbH}$ ) and the measurements were repeated at least five times. After a 25-min dark adaptation period, OJIP transients were induced by red light $(650 \mathrm{~nm})$ with a $3000 \mu \mathrm{mol}$ photons $\mathrm{m}^{-2} \mathrm{~s}^{-1}$ light intensity and recorded by the instrument. The OJIP transient data were analyzed using the JIP-test (Chen et al., 2013). The indexes of $\varphi_{\text {Po }}$ (maximum quantum yield for primary photochemistry, namely $F_{\mathrm{V}} / F_{\mathrm{M}}$ ), $\Psi_{\mathrm{E} 0}$ (efficiency/probability with which a PSII trapped electron is transferred from $\mathrm{Q}_{\mathrm{A}}$ to $\mathrm{Q}_{\mathrm{B}}$ ), $\delta_{\mathrm{R} 0}$ (efficiency/probability with which an electron from $\mathrm{Q}_{\mathrm{B}}$ is transferred to PSI acceptors), ABS/RC (absorbed photon flux per RC), $\gamma_{\mathrm{RC}}$ (probability that a PSII Chl molecule functions as 
a $\mathrm{RC}$ ), $\mathrm{PI}_{\mathrm{ABS}}$ (performance index for energy conservation from photons absorbed by PSII antenna, for the reduction of $\mathrm{Q}_{\mathrm{B}}$ ) and $\mathrm{PI}_{\text {Total }}$ (performance index for energy conservation from photons absorbed by PSII antenna until reduction of PSI acceptors) were employed in to study the energy flow and photosynthetic activities of tall fescue leaves.

\section{Preparation of Thylakoid Membranes}

The preparation of thylakoid membranes refers to the methods mentioned by Robinson (Robinson and Yocum, 1980) with slight modification. Briefly, $30 \mathrm{~g}$ of tall fescue leaves was crushed within $50 \mathrm{~mL}$ of precooled $0.4 \mathrm{M} \mathrm{NaCl}, 2 \mathrm{mM} \mathrm{MgCl}, 0.2 \%$ bovine serum albumin (BSA), and $20 \mathrm{mM}$ Tricine ( $\mathrm{pH}$ 8.0) solution by a Waring blender. The homogenate was then filtered through four layers of cheesecloth, and centrifuged at $4^{\circ} \mathrm{C}$, and $500 \mathrm{~g}$ for $3 \mathrm{~min}$. The supernatant was then centrifuged at $4^{\circ} \mathrm{C}$, and $4000 \mathrm{~g}$ for $10 \mathrm{~min}$ to collect broken chloroplasts. The pellets were resuspended in $10 \mathrm{~mL}$ of precooled $0.1 \mathrm{M} \mathrm{NaCl}, 5 \mathrm{mM} \mathrm{MgCl}_{2}$, $0.2 \% \mathrm{BSA}$, and $20 \mathrm{mM}$ Tricine ( $\mathrm{pH} 8.0$ ) solution, and centrifuged at $4^{\circ} \mathrm{C}$, and $4000 \mathrm{~g}$ for $10 \mathrm{~min}$ twice; the pellet was then washed with the solution mentioned above. Finally, thylakoid membranes were suspended in precooled $0.4 \mathrm{M}$ sucrose, $15 \mathrm{mM}$ $\mathrm{NaCl}, 0.2 \% \mathrm{BSA}$, and $20 \mathrm{mM}$ Tricine ( $\mathrm{pH} 8.0$ ) solution. The thylakoid concentration was adjusted to $200 \mu \mathrm{g} \mathrm{Chl} / \mathrm{mL}$ before subsequent treatment.

\section{Regeneration of the K-Step of Tall Fescue Thylakoid Membranes}

Tall fescue thylakoids were incubated with water $(\mathrm{Ct})$ or $20 \mathrm{mM}$ ASC (Asc) in a $44^{\circ} \mathrm{C}$ water bath for $1 \mathrm{~min}$ to inactivate OEC. Then, $20 \mu \mathrm{L}$ of thylakoids $(200 \mu \mathrm{g} \mathrm{Chl} / \mathrm{mL})$ was dropped onto a nitrocellulose membrane, and after 25 min of dark adaption, $\mathrm{Chl} a$ fluorescence transients were induced and recorded by two 5-ms saturated pulses that were spaced $200 \mathrm{~ms}$ apart.

\section{Determination of ASC}

Tall fescue leaves were crushed in liquid nitrogen, and 0.05-g samples were added to $1 \mathrm{~mL}$ of perchloric acid, shaken vigorously, and centrifuged at $4^{\circ} \mathrm{C}$, and $14000 \mathrm{~g}$ for $15 \mathrm{~min}$. Then, $400 \mu \mathrm{L}$ of supernatant was mixed with $65 \mu \mathrm{L}$ of a $0.1 \mathrm{M}$ HEPES-KOH $(\mathrm{pH}=7)$ solution, and $98 \mu \mathrm{L}$ of $1.7 \mathrm{M} \mathrm{K}_{2} \mathrm{CO}_{3}$ was added to adjust the $\mathrm{pH}$ to $4-5$. The mixture was centrifuged at $4^{\circ} \mathrm{C}$, and $14000 \mathrm{~g}$ for $15 \mathrm{~min}$, and $50 \mu \mathrm{L}$ of supernatant was mixed with $940 \mu \mathrm{L}$ of $60 \mathrm{mM}$ phosphate buffer $(\mathrm{pH}=6.3)$. Then, 1 unit of ascorbate peroxidase (APX, Sigma, St. Louis, MO, United States) was added to the above mixture, and the absorbance at $265 \mathrm{~nm}$ after 2 minutes was recorded. The content of ASC was calculated by use of a standard curve.

\section{Determination of Hydrogen Peroxide and Superoxide Radical}

The hydrogen peroxide and superoxide radical contents were measured by histological staining method (Ramel et al., 2009). Tall fescue leaves were immersed with $1 \mathrm{mg} \mathrm{mL}^{-1} \mathrm{DAB}\left(3,3^{\prime}\right.$ diamino-benzidine, Sigma, St. Louis, MO, United States) staining solution (hydrochloric acid, pH 3.8) for $30 \mathrm{~min}$. Stained leaves were then bleached in an acetic acid-glycerol-ethanol (1/1/3) $(\mathrm{v} / \mathrm{v} / \mathrm{v})$ solution at $100^{\circ} \mathrm{C}$ for $5 \mathrm{~min}$, and stored in a glycerolethanol (1/4) (v/v) solution for $30 \mathrm{~min}$. Next, the leaves were ground in liquid nitrogen, homogenized in $0.2 \mathrm{M} \mathrm{HClO}_{4}$, and centrifuged for $10 \mathrm{~min}$ at $12000 \mathrm{~g}$. A450 was immediately measured and compared with a standard curve containing known amounts of $\mathrm{H}_{2} \mathrm{O}_{2}$ in a $0.2 \mathrm{M} \mathrm{HClO}_{4}$-DAB solution.

In the case of superoxide radical $\left(\mathrm{O}_{2}{ }^{\bullet-}\right)$ determination, leaves were immersed and infiltrated with $2 \mathrm{mg} \mathrm{mL}^{-1}$ nitroblue tetrazolium (NBT, Sigma, St. Louis, MO, United States) staining solution (25 mM KOH-HEPES buffer, $\mathrm{pH} 7.8$ ) and incubated in the dark for $30 \mathrm{~min}$. The stained leaves were bleached in an acetic acid-glycerol-ethanol $(1 / 1 / 3)(\mathrm{v} / \mathrm{v} / \mathrm{v})$ solution at $100^{\circ} \mathrm{C}$ for $5 \mathrm{~min}$ and stored in a glycerol-ethanol (1/4) (v/v) solution for $30 \mathrm{~min}$. Then, the NBT-stained leaves were ground in liquid nitrogen, and the formazan content in the obtained powder was solubilized in $2 \mathrm{M} \mathrm{KOH}-\mathrm{DMSO}(1 / 1.16)(\mathrm{v} / \mathrm{v})$, and centrifuged for $10 \mathrm{~min}$ at $12000 \mathrm{~g}$. The A630 of the solution was immediately measured and compared with a standard curve obtained from known amounts of NBT.

\section{APX Activities}

Tall fescue leaves were crushed in liquid nitrogen, and crude enzymes were extracted by $50 \mathrm{mM}$ phosphate buffer $(\mathrm{pH}=7.8)$. After centrifuging at $4^{\circ} \mathrm{C}$, and $12000 \mathrm{~g}$ for $15 \mathrm{~min}$, the supernatant was used to determine the APX activity following the method introduced by Nakano (Nakano and Asada, 1981). Briefly, the reaction solution contained $50 \mathrm{mM}$ acetate $(\mathrm{pH}=5.8), 5 \mathrm{mM}$ $\mathrm{H}_{2} \mathrm{O}_{2}, 10 \mathrm{mM}$ Asc, and $3 \mu \mathrm{M}$ EDTA. After the addition of the enzyme supernatant, the absorbance at $290 \mathrm{~nm}$ was recorded for 2 min with $10 \mathrm{~s}$ intervals, and 1 unit of APX activity was defined as the decrease of 0.01 absorbance per minute.

\section{Quantitative RT-PCR Analysis}

Total RNA was isolated and purified from treated leaves according to the protocol of RNeasy ${ }^{\circledR}$ plant mini kits (Qiagen). The expressions of selected genes were analyzed by real-time quantitative reverse transcriptase (RT-PCR) using the fluorescent intercalating dye SYBRGreen with a detection system (Opticon 2, MJ Research, Waltham, MA, United States). The VTC2 coding mRNA sequence was amplified with the following primers: forward (5'-ACCTTCACAGGAGGATGCTG-3') and reverse $\left(5^{\prime}\right.$-CCACGCGGAACTCTGTAGGG-3'). The VTC4 coding mRNA sequence was amplified with the following primers: forward (5'-GACCACAAGTTCATCGGCGAG-3') and reverse (5'-CCACGAACAGCTGTGAAAAGCT-3') (Hu et al., 2014). eEF1A (s) was used as a standard control for RT-PCR. The twostep RT-PCR procedure was performed following the methods we described earlier.

\section{Statistical Analysis}

Each experiment was repeated at least five times. The values in the figures or tables are provided as the mean \pm SD. Statistical analyses were performed by Duncan's multiple range tests or twoway ANOVA as indicated in the figures or tables. Different letters in the figures or tables indicate statistically significant differences at $P<0.05$. 


\section{RESULTS}

\section{Effects of Asc on Tall Fescue OJIP Transients against Heat Stress}

Tall fescue leaves were subjected to heat stress and Asc treatment to study the role of Asc in PSII against heat stress using the OJIP fluorescence transient method. The fluorescence transient curves are shown in Figure 1. From the figure, heat stress treatment significantly affected the OJIP fluorescence transient curves. After heat stress, higher initial fluorescence as well as lower maximum fluorescence were observed. Additionally, the clear $\mathrm{K}$ step of heat-stress treated samples was observed at $0.3 \mathrm{~ms}$, which indicates thermal damage to the PSII. Additionally, compared 44Ct curve, the 44Asc curve showed a lower K step, which indicates that Asc treatment alleviated the damage from heat stress. To better understand the effect of heat stress and Asc treatment on the $\mathrm{K}$ step, K-band, and L-band of OJIP curves were studied and are shown in Figure 2. Figures 2A,B depict the relative variable fluorescence between $F_{0}$ and $F_{\mathrm{K}}$ ( $\mathrm{W}_{\mathrm{OK}}$ ) as well as the differences of transients in samples treated with heat stress and Asc minus the room temperature transient (24Ct) $\left(\Delta \mathrm{W}_{\mathrm{OK}}\right)$. In Figure $2 \mathbf{B}$, the sequence from upper to lower was $44 \mathrm{Ct}, 44 \mathrm{Asc}, 24 \mathrm{Ct}$, and $24 \mathrm{Asc}$, which indicates heat stress damage on PSII and the positive role of Asc in this process.

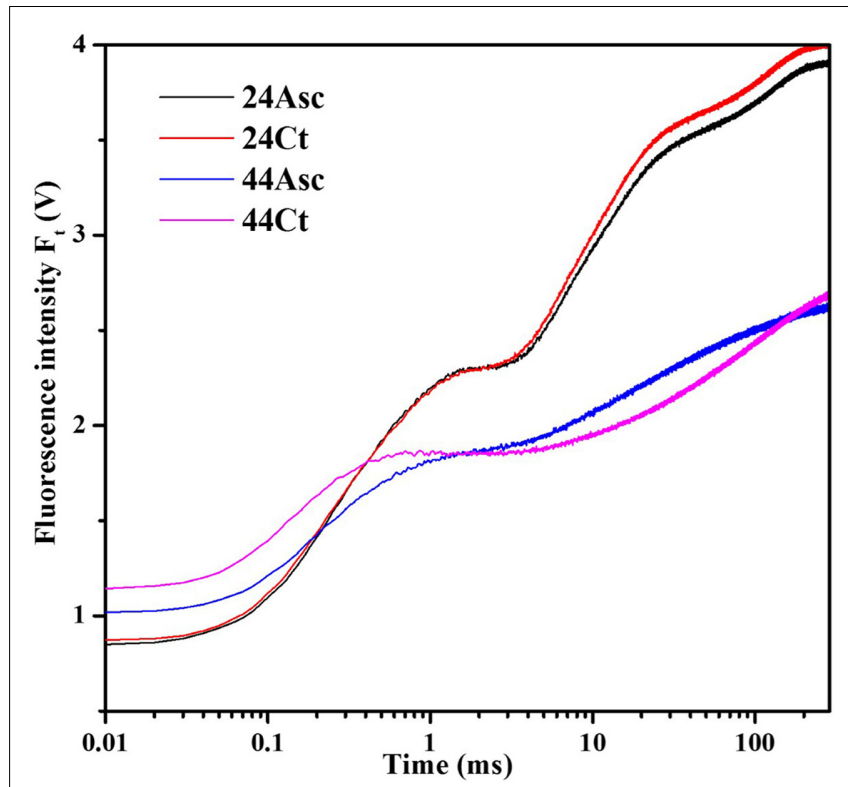

FIGURE 1 | Effects of heat stress and L-Ascorbate (Asc) on OJIP fluorescence transients in tall fescue leaves. After the reagent treatments, the petiole of the leaves was immersed $1 \mathrm{~cm}$ deep within distilled water kept in a falcon tube with Asc (24Asc and 44Asc) or not (24Ct and 44Ct). Then the tubes were placed in a growth cabinet with the temperature of $24^{\circ} \mathrm{C}(24 \mathrm{Ct}$ and $24 \mathrm{Asc}$ ) or $44^{\circ} \mathrm{C}$ (44Ct and 44Asc), and light intensity of $100 \mu \mathrm{mol}$ photons $\mathrm{m}^{-2} \mathrm{~s}^{-1}$, and a humidity of $80 \%$ for $4 \mathrm{~h}$. Then after a $25-\mathrm{min}$ dark-adaption period at $24^{\circ} \mathrm{C}$, the OJIP curves were induced by a red light provided by a PAM 2500. Chl a fluorescence emissions were recorded by the instrument and the most typical curves are shown here.
The basic fluorescence parameters were extracted from the curves and are provided in Table 1. The JIP-test was used to further understand the energy flow and electron transport in PSII in this study, and the relevant data are provided in Table 2. Compared to $24 \mathrm{Ct}, 44 \mathrm{Ct}$ had lower values of $\varphi_{\mathrm{Po}}, \gamma_{\mathrm{RC}}$ and $\mathrm{PI}_{\mathrm{ABS}}$ as well as higher values of $\delta_{\mathrm{R} 0}$ and $\mathrm{ABS} / \mathrm{RC}$. Additionally, compared to $44 \mathrm{Ct}$, $44 \mathrm{Asc}$ had lower values of $\Psi_{\mathrm{E} 0}, \delta_{\mathrm{R} 0}, \mathrm{ABS} / \mathrm{RC}$, and $\mathrm{PI}_{\text {Total }}$ as well as higher values of $\varphi_{\mathrm{Po}}, \gamma_{\mathrm{RC}}$, and $\mathrm{PI}_{\mathrm{ABS}}$.

\section{Effect of Heat Stress and Asc Treatments on ROS Production in Tall Fescue Leaves}

To better understand the role of Asc in tall fescue under heat stress, the ROS content of tall fescue leaves subjected to the heat stress and Asc treatment were studied in Figure 3. From the

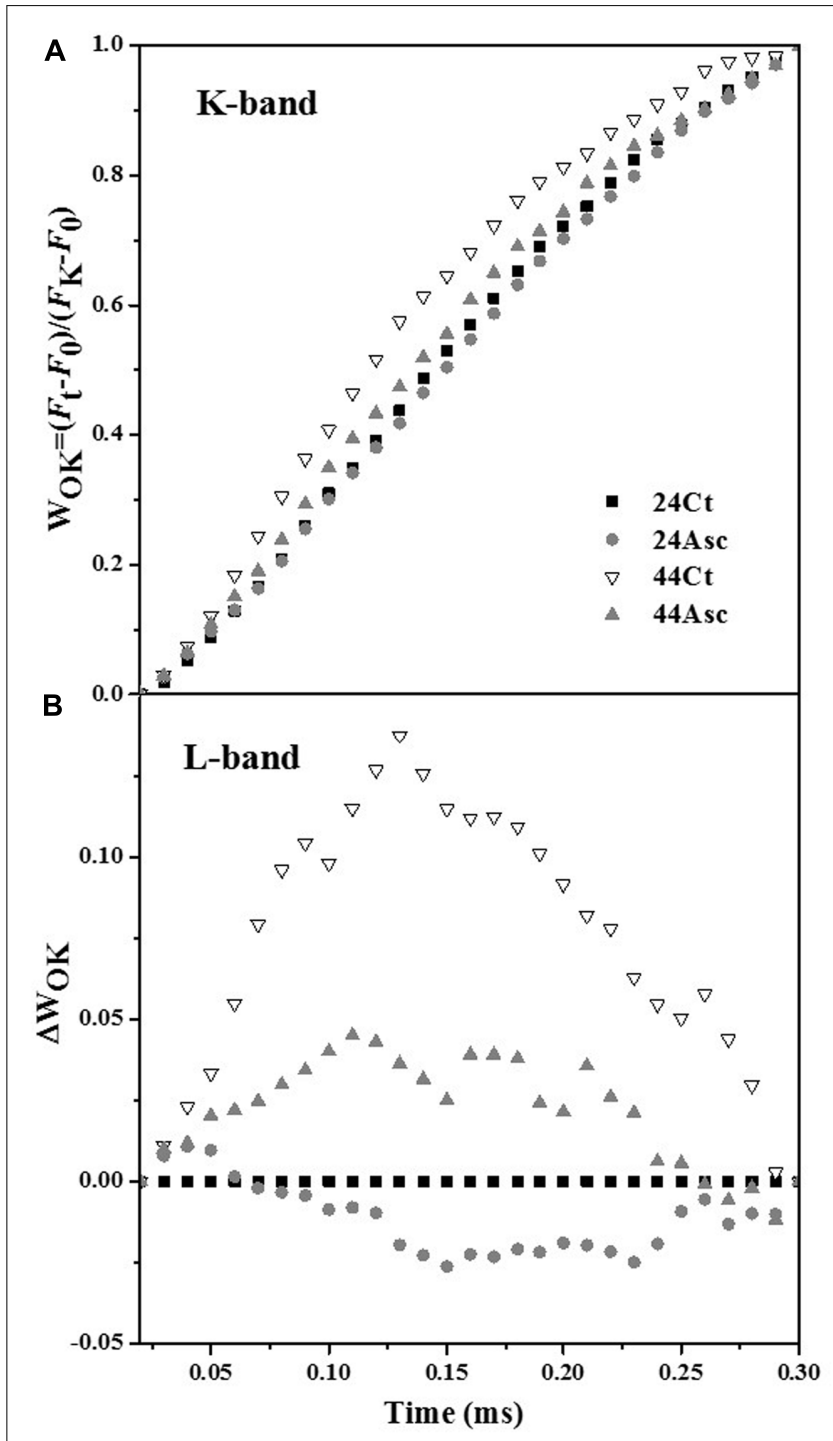

FIGURE 2 | Effects of heat stress and Asc on the K-band and L-band in tall fescue leaves. The differences in the heat stress and Asc treatment samples $\left(\boldsymbol{\Delta} \bigvee_{\mathrm{t}}\right),(\mathbf{A})$ between $F_{0}$ and $F_{\mathrm{K}}: W_{\mathrm{OK}}=\left(F_{\mathrm{t}}-F_{0}\right) /\left(F_{\mathrm{K}}-F_{0}\right)$ and $\mathbf{( B )}$ the differences of the heat stress samples to the control sample $(24 \mathrm{Ct})\left(\Delta \mathrm{W}_{\mathrm{OK}}\right)$. 
TABLE 1 | Basic photosynthetic parameters extracted from the OJIP transient curves.

\begin{tabular}{|c|c|c|c|c|}
\hline Parameters & $24 \mathrm{Ct}$ & 24Asc & $44 \mathrm{Ct}$ & 44Asc \\
\hline$F_{0}$ & $0.88 \pm 0.10$ & $0.86 \pm 0.10$ & $1.16 \pm 0.14$ & $1.03 \pm 0.13$ \\
\hline$F_{300}$ & $1.65 \pm 0.21$ & $1.65 \pm 0.18$ & $1.74 \pm 0.23$ & $1.56 \pm 0.2$ \\
\hline$F_{\mathrm{J}}$ & $2.29 \pm 0.32$ & $2.31 \pm 0.29$ & $1.85 \pm 0.24$ & $1.86 \pm 0.24$ \\
\hline$F_{1}$ & $3.56 \pm 0.45$ & $3.46 \pm 0.44$ & $2.14 \pm 0.28$ & $2.3 \pm 0.31$ \\
\hline$F_{\mathrm{M}}$ & $4.07 \pm 0.54$ & $3.94 \pm 0.51$ & $2.72 \pm 0.31$ & $2.66 \pm 0.35$ \\
\hline
\end{tabular}

figure, compared to $24 \mathrm{Ct}$, the additional $20 \mathrm{mM}$ Asc treatment (24Asc) decreased the content of $\mathrm{H}_{2} \mathrm{O}_{2}$ and $\mathrm{O}_{2}{ }^{\bullet-}$ of tall fescue leaves by approximately 16 and $48 \%$, respectively. The heat stress treatment (44Ct) increased the content of $\mathrm{H}_{2} \mathrm{O}_{2}$ and $\mathrm{O}_{2}{ }^{\bullet-}$ of leaves by approximately 2.1 - and 2.5 -fold than those of tall fescue under room temperature $(24 \mathrm{Ct})$, respectively. After addition of $20 \mathrm{mM}$ Asc (44Asc), the content of $\mathrm{H}_{2} \mathrm{O}_{2}$ and $\mathrm{O}_{2}{ }^{\bullet-}$ of tall fescue leaves under heat stress decreased by approximately 28 and 56\%, respectively.

\section{Alternative Electron Donor Studies of Tall Fescue Thylakoids}

To better understand alternative electron donor of tall fescue PSII, tall fescue thylakoids were extracted and incubated at $44^{\circ} \mathrm{C}$ for $1 \mathrm{~min}$ to inactivate OEC, and Asc was used to study exogenous electron donors in PSII (Figure 4). From the figure, regeneration of the K-step as a function of the dark interval between two 5$\mathrm{ms}$ saturated pulses was studied. Compared to the control (Ct), an additional $20 \mathrm{mM}$ Asc treatment (Asc) peaked the Chl $a$ fluorescence transient curves at $0.3 \mathrm{~ms}$.

\section{Effects of the Heat Stress Treatment on the APX Activities, Asc Contents, and Transcription Levels of VTC4 and VTC2}

To further understand the regulation of Asc synthesis for tall fescue leaves against heat stress, the APX activity and Asc content were studied and are shown in Figure 5. In Figure 5, after heat stress, the tall fescue APX activity (44Ct) and Asc content (44Ct) increased significantly. Additionally, the expression profiles of VTC2 and VTC4 were studied, and the data are shown in Figure 6. After the heat stress treatment (44Ct), the transcription levels of VTC2 and VTC4 significantly decreased.

\section{DISCUSSION}

Even though the tall fescue has considerable heat tolerance, heat stress limits its growth and development in transitional and warm climatic regions (Sun et al., 2015). In this study, the effects of exogenous Asc on the tall fescue against heat stress and regulation of Asc synthesis of tall fescue under heat stress have been studied. On the electron donor side, heat stress treatment increased initial fluorescence $\left(\mathrm{F}_{0}\right)$ of tall fescue leaves, as shown in Figure 1. After dark adaption, all of the primary quinone acceptors in PSII are in an oxidized state, and $\mathrm{F}_{0}$ is the level of fluorescence emission of PSII induced by weak illumination. $\mathrm{F}_{0}$ refers to the concept of open, close and inactivation of the RC in PSII (Tsimilli-Michael and Strasser, 2008). As shown in Table 2, heat stress treatment significantly decreased the value of $\gamma_{\mathrm{RC}}$ (44Ct versus $24 \mathrm{Ct}$ ), which indicates that heat stress treatment inactivated the RC in PSII. This response may be related to the aggregation of Light Harvest Complex II (Tang et al., 2007) or increase in the $\mathrm{Q}_{B}$ non-reducing center (Mehta et al., 2010). The K-step ( $0.3 \mathrm{~ms}$ ) observed in $44 \mathrm{Ct}$ means that after heat stress, the connection between RC and OEC decreased, and electron transport from OEC to the tyrosine residue $\left(\operatorname{Try}_{z}{ }^{+}\right.$, D1-Tyr161) was inhibited. Figure 2 explores the K-band and L-band of the OJIP curves of tall fescue leaves under room temperature and heat stress. Heat stress treatment significantly increased the amplitude of the L-band (44Ct versus $24 \mathrm{Ct}$ ), indicating that after heat stress treatment, the PSII units of tall fescue leaves were less grouped or that less energy was exchanged between independent PSII units, namely, there was a lose of stability and an increase in fragility.

In this study, the JIP test was used to study the energy flow of PSII of tall fescue leaves subjected to heat stress treatment. The JIP test evaluated the three major steps that occurred in electron transport in photosystem, namely, trapping photons, electron transport after $\mathrm{Q}_{\mathrm{A}}{ }^{-}$to intersystem electron acceptors and reduction of end acceptors (Stirbet and Govindjee, 2011), as well as the energy flow efficiency of these three steps, which was reflected through the values of $\varphi_{\mathrm{Po}}, \Psi_{\mathrm{E} 0}$, and $\delta_{\mathrm{R} 0}$. After

TABLE 2 | Photosynthetic parameters deduced by the JIP test analysis of fluorescence transients.

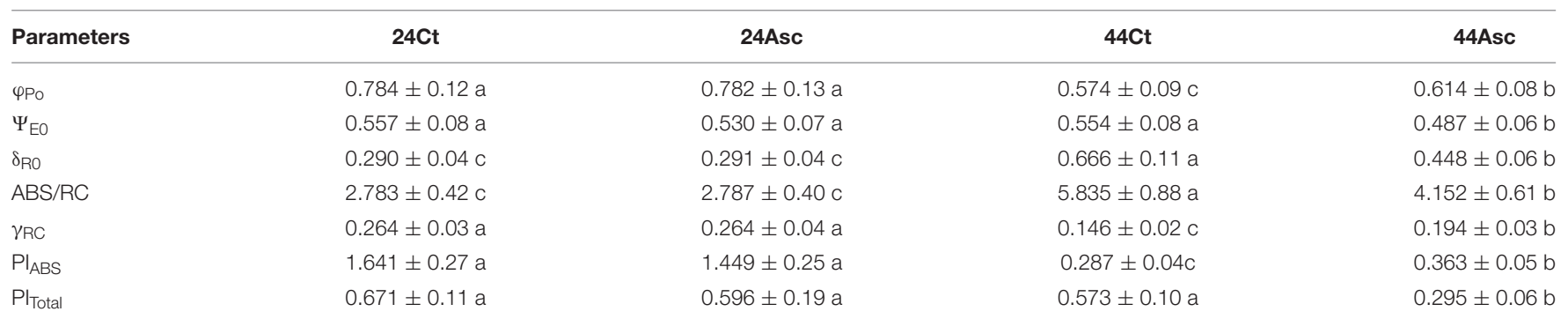

Subscript " $O$ " indicates that the parameter refers to the onset of illumination. Values are given as the means $\pm S D(n=5)$, and different letters indicate statistically significant differences at $P<0.05$. 


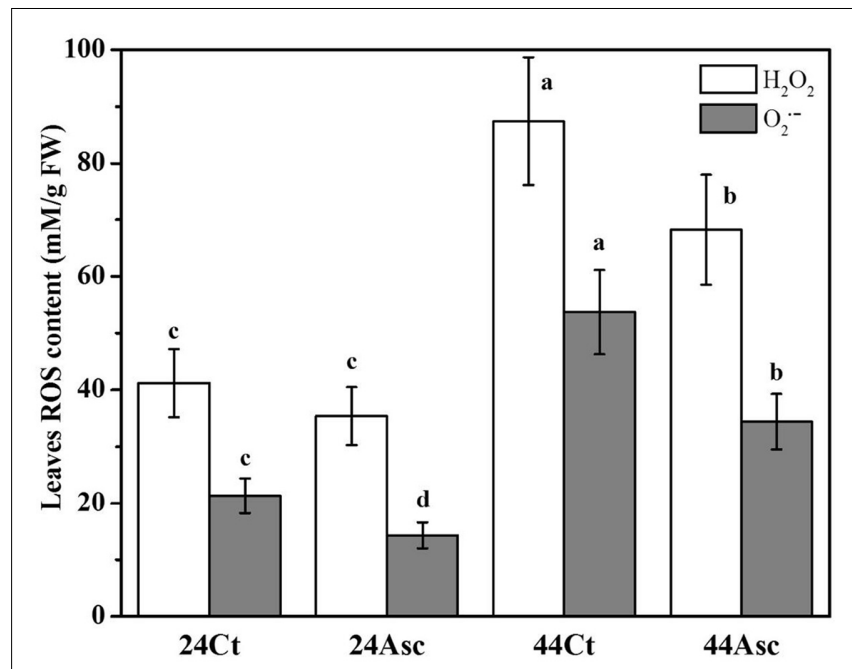

FIGURE 3 | Effect of heat stress and Asc on ROS production in tall fescue leaves. Values are given as the means $\pm \operatorname{SD}(n=5)$, and two-way ANOVA was used to study the differences between heat stress and Asc treatments. Different letters indicate statistically significant differences at $P<0.05$. heat stress treatment, we observed a lower value of $\varphi_{\text {Po }}$ and a higher value of $\delta_{\mathrm{R} 0}$, and in this study, there was no significant difference between the values of $\Psi_{E}$ under room temperature and heat stress treatment. Thus, heat stress treatment inhibited the activities of PSII in the photochemical phase of the OJIP curves and the activity of PSII in the thermal phase of the OJIP curves was elevated.

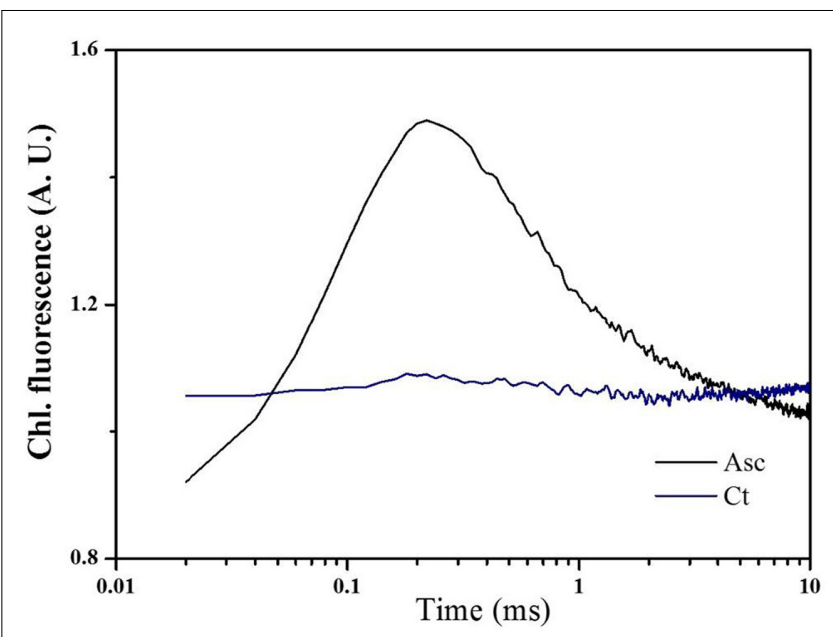

FIGURE 4 | Effects of Asc on the electron donor side of PSII under heat stress. Tall fescue thylakoids were incubated with water (Ct) or $20 \mathrm{mM}$ Asc (Asc) in a $44^{\circ} \mathrm{C}$ water bath for $1 \mathrm{~min}$ to inactivate the oxygen evolution complex. Then, $20 \mu \mathrm{L}$ of thylakoids $(200 \mu \mathrm{g} \mathrm{Chl} / \mathrm{mL}$ ) was dropped onto a nitrocellulose membrane, and after a 20-min dark adaption period, Chl a fluorescence transients were induced and recorded by two 5-ms saturated pulses that were spaced $200 \mathrm{~ms}$ apart.

Asc is a multifunctional metabolite in plants and plays an important role in plants against abiotic stresses. In this research, the multiple roles of Asc in tall fescue against heat stress were studied. Additionally, $20 \mathrm{mM}$ Asc was used to incubate tall fescue leaves before heat stress. Addition of Asc facilitated the
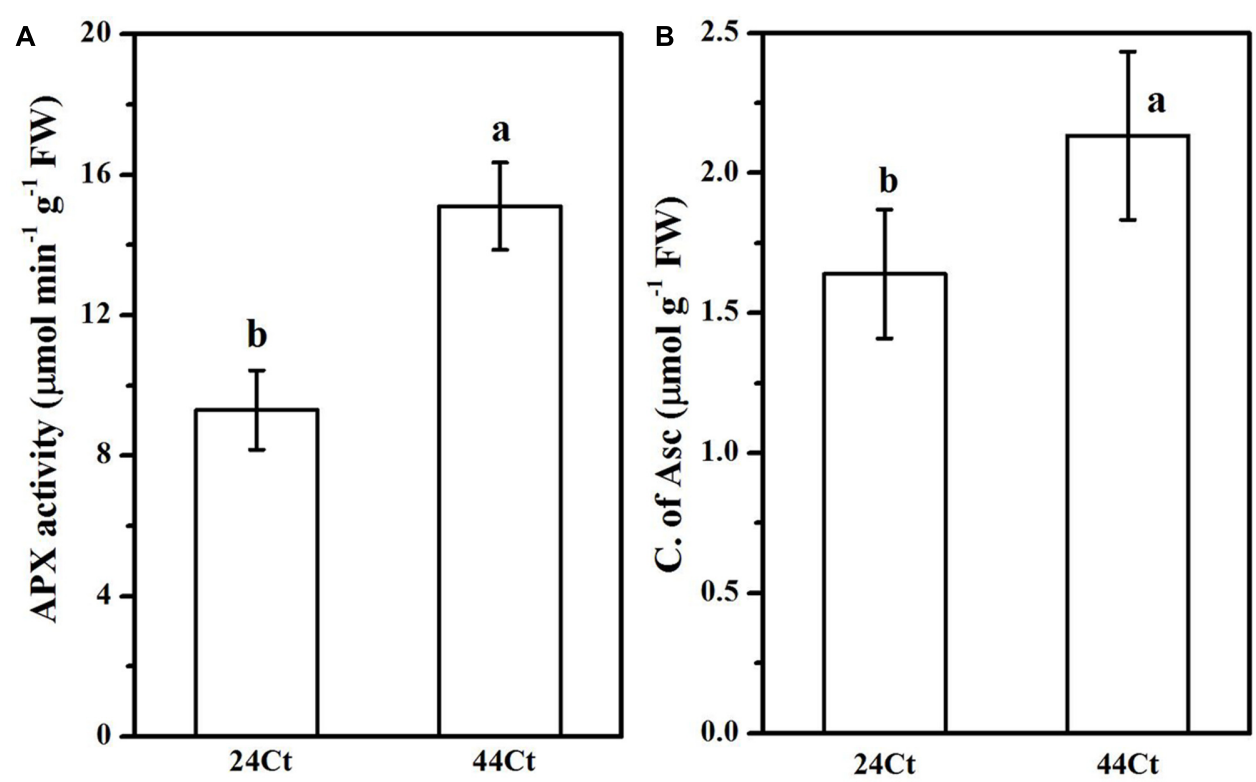

FIGURE 5 | Effects of heat stress on the APX activity (A) and production of ASC (B). Tall fescue leaves were vacuum infiltrated with water (24Ct, 44 Ct) for 15 min in the dark. Then, they were moved into a growth cabinet with a temperature of 24 or $44^{\circ} \mathrm{C}$, a light intensity of $100 \mu \mathrm{mol}$ photons $\mathrm{m}^{-2} \mathrm{~s}^{-1}$ and a humidity of $80 \%$ for $4 \mathrm{~h}$. Afterward, tall fescue leaves were ground in liquid nitrogen and used to determine the concentration of Asc as well as APX activity. Values are provided as the means $\pm \mathrm{SD}(n=5)$, and different letters indicate statistically significant difference at $P<0.05$ among the treatments according to Duncan's multiple range tests. 


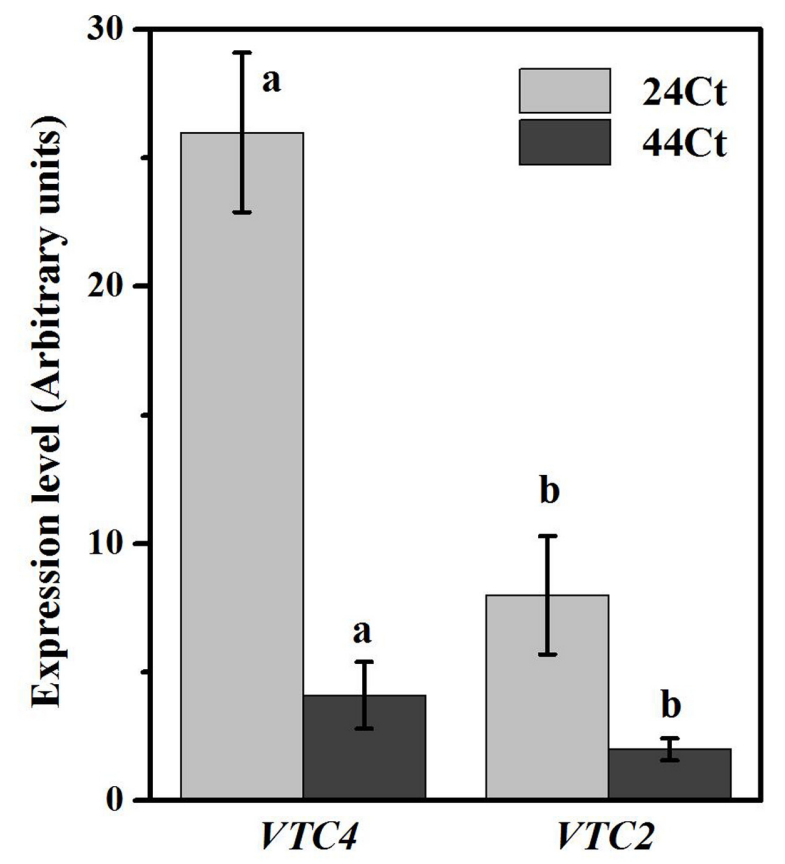

FIGURE 6 | Effects of heat stress on the Asc-related gene expression. Tall fescue leaves were vacuum infiltrated with water (24Ct, 44Ct) for $15 \mathrm{~min}$ in the dark. Then, they were moved into a growth cabinet with a temperature of 24 or $44^{\circ} \mathrm{C}$, a light intensity of $100 \mu \mathrm{mol}$ photons $\mathrm{m}^{-2} \mathrm{~s}^{-1}$ and a humidity of $80 \%$ for $4 \mathrm{~h}$. Afterward, tall fescue leaves were ground in liquid nitrogen and used to study mRNA transcription. Values are given as the means $\pm \mathrm{SD}$ $(n=5)$, and different letters indicate statistically significant difference at $P<0.05$ among the treatments from Duncan's multiple range tests.

behaviors of the photosystem. In the photochemical phase, the addition of Asc reduced the amplitude of the K-band at room temperature (Figure 2A, 24Ct versus 24Asc) and under heat stress (Figure 2A, 44Ct versus 44Asc). Because the amplitude of the K-band corresponds to electron transport from OEC to the $\mathrm{Try}_{\mathrm{z}}{ }^{+}$(Tsimilli-Michael and Strasser, 2008), the addition of Asc facilitated electron transport to $\mathrm{Try}_{z}{ }^{+}$.

That may because, firstly, Asc could scavenge ROS, both enzymatically and nonenzymatically in the chloroplast stromas (Tsimilli-Michael and Strasser, 2008). High ROS produced in a water-oxidation reaction are extremely destructive to the D1 protein, and heat stress treatment could enhance the susceptibility of this photosynthetic apparatus (Takahashi et al., 2004). The addition of $20 \mathrm{mM}$ Asc significantly decreased the level of ROS in tall fescue leaves under room temperature as well as heat stress (Figure 3), which alleviated the toxicity of ROS to the $\mathrm{D} 1$ protein and improved the behavior of electron transport from OEC to $\mathrm{Try}_{z}{ }^{+}$. The decreased ROS content in 44Asc may have also contributed to the recovery of RC in PSII, as shown in the increased value of $\gamma_{\mathrm{RC}}$ in Table 2 (44Asc versus $44 \mathrm{Ct}$ ).

Secondly, when the activity of OEC was inhibited under heat stress treatment, Asc could acted as an alternative electron donor of water to maintain the electron transport chain in PSII (Toth et al., 2009, 2011). In this study the role of Asc as an alternative electron donor of tall fescue thylakoids was verified in Figure 4 through regeneration of the $\mathrm{K}$ step of the thylakoids after two 5ms short saturating pulses separated by $200 \mathrm{~ms}$ dark durations. The treatment of thylakoids at $44^{\circ} \mathrm{C}$ for $1 \mathrm{~min}$ would inactive the function of OEC of splitting water as well as providing electron to $\operatorname{Try}_{\mathrm{z}}{ }^{+}$. In this case, the first $5 \mathrm{~ms}$ short saturating pulse would consume electrons donated by $\mathrm{Tyr}_{\mathrm{z}}$ and form $\mathrm{Try}_{\mathrm{z}}{ }^{+}$, and rereduction of $\mathrm{Tyr}_{\mathrm{z}}{ }^{+}$after $200 \mathrm{~ms}$ was inhibited due to the impairment of OEC as well as lose of alternative electron donor in thylakoid prepartion procedure (Toth et al., 2009). Thus the second $5 \mathrm{~ms}$ short saturating pulse (at $200 \mathrm{~ms}$, after the first $5 \mathrm{~ms}$ short saturating pulse ) could not induce signal as shown in $\mathrm{Ct}$ in Figure 4. However, the addition of Asc induce a clear peak at $0.3 \mathrm{~ms}$, which suggests that in tall fescue Asc could serve as an alternative electron donor to provide electrons to PSII via Tryz ${ }^{+}$ (Srivastava et al., 1997; Toth et al., 2011).

It has been widely accepted that under heat stress, the photochemical phase of the photosystem is inhibited due to inactivation of RC or damage to OEC, and so on. Due to the roles of Asc in regulating ROS and serving as an alternative electron donor in PSII, the performance of the photochemical phase of tall fescue treated with Asc should be largely elevated, which was demonstrated by the increased values of $\varphi_{\mathrm{Po}}$ and $\gamma_{\mathrm{RC}}$ of tall fescue leaves pretreated with $20 \mathrm{mM}$ Asc before heat stress treatment (44Asc versus 44Ct, Table 2). Additionally, we observed that, under heat stress, after pretreatment with Asc, the value of $\delta_{\mathrm{R} 0}$ decreased by approximately $48.6 \%$, which indicated that there was lower activity of the thermal phase in the photosystem. Havaux (1996) reported that the PSI of potato leaves could catalyze an electron flow from reductants against heat-stress inhibited PSII activities, and the elevated activities of the thermal phase of the photosystem may be an important strategy for plants to regulate their energy flow in the photosystem to respond to heat stress. However, our previous studies showed that the heat tolerant tall fescue genotype under heat stress treatment had a relatively lower value of $\delta_{R 0}$ (Chen et al., 2014) compared to the heat sensitive genotype, which correlates well with the results obtained in this study. Thus, in tall fescue, the increased activity of PSII in the thermal phase of the OJIP curves may be harmful, and the lower activity of the PSII may contribute to limiting the energy input into the photosystem as well as limiting the production of ROS to resist the heat stress. This result was also in line with our findings, in which pretreatment with Asc significantly decreased the values of $\mathrm{PI}_{\text {Total }}$ under heat stress (Table 2).

Regulation of APX activity and Asc synthesis of tall fescue under the heat stress treatment were also studied. Heat stress significantly increased the APX activity and Asc content in the tall fescue leaves. We observed that the transcription level of VTC4 and VTC2 decreased after the heat stress treatment. The VTC2 gene is the last missing enzyme in the L-galactose pathway in Asc synthesis in higher plants (Linster and Clarke, 2008), and VTC2 encods GDP-L-galactose phosphorylase, which is the first committed step in Asc synthesis. The transcription level of VTC2 in Arabidopsis decreases with supplementation of Asc, which suggests that there is feedback inhibition in Asc synthesis in plants (Dowdle et al., 2007). Additionally, heat stress decreases VTC2 transcripts in kiwifruit leaves (Li et al., 2013). 
The VTC4 encoded L-galactose 1-phosphate phosphatase (GPP) is a bifunctional enzyme that affects myoinositol and Asc synthesis in plants (Torabinejad et al., 2009), and its expression level is reduced after exposure to heat stress in tomato leaves. These findings agree with the results we obtained in this study and suggest that in tall fescue, heat stress induces the synthesis of Asc, which inhibits genes transcription of the Asc synthesis pathway through feedback control. Thus, the regulation of elevated Asc after heat stress may occur at other levels, for example, histone modification and phosphorylation of target enzymes, which requires further study.

\section{CONCLUSION}

In this study, heat stress treatment inhibited the activity of the photochemical phase of PSII and elevated the activity of the thermal phase of the photosystem of tall fescue. The addition of Asc facilitated the behavior of the photochemical phase of the PSII by reducing the ROS content as well as acting as an alternative electron donor to provide electron to the tyrosine residue of the D1 protein. Additionally, exogenous Asc reduced

\section{REFERENCES}

Attallah, C. V., Welchen, E., and Gonzalez, D. H. (2007). The promoters of Arabidopsis thaliana genes AtCOX17-1 and -2, encoding a copper chaperone involved in cytochrome c oxidase biogenesis, are preferentially active in roots and anthers and induced by biotic and abiotic stress. Physiol. Plant. 129, 123-134. doi: 10.1111/j.1399-3054.2006.00776.x

Barra, M., Haumann, M., and Dau, H. (2005). Specific loss of the extrinsic $18 \mathrm{KDa}$ protein from photosystem II upon heating to 47 degrees $\mathrm{C}$ causes inactivation of oxygen evolution likely due to Ca release from the Mn-complex. Photosynth. Res. 84, 231-237. doi: 10.1007/s11120-004-7158-x

Chen, K., Chen, L., Fan, J., and Fu, J. (2013). Alleviation of heat damage to photosystem II by nitric oxide in tall fescue. Photosynth. Res. 116, 21-31. doi: 10.1007/s11120-013-9883-5

Chen, K., Sun, X., Amombo, E., Zhu, Q., Zhao, Z., Chen, L., et al. (2014). High correlation between thermotolerance and photosystem II activity in tall fescue. Photosynth. Res. 122, 305-314. doi: 10.1007/s11120-014-0035-3

Debolt, S., Melino, V., and Ford, C. M. (2007). Ascorbate as a biosynthetic precursor in plants. Ann. Bot. 99, 3-8. doi: 10.1093/aob/mcl236

Dowdle, J., Ishikawa, T., Gatzek, S., Rolinski, S., and Smirnoff, N. (2007). Two genes in Arabidopsis thaliana encoding GDP-L-galactose phosphorylase are required for ascorbate biosynthesis and seedling viability. Plant J. 52, 673-689. doi: 10.1111/j.1365-313X.2007.03266.x

Ederli, L., Morettini, R., Borgogni, A., Wasternack, C., Miersch, O., Reale, L., et al. (2006). Interaction between nitric oxide and ethylene in the induction of alternative oxidase in ozone-treated tobacco plants. Plant Physiol. 142, 595-608. doi: $10.1104 /$ pp.106.085472

Essemine, J., Govindachary, S., Ammar, S., Bouzid, S., and Carpentier, R. (2011). Abolition of photosystem I cyclic electron flow in Arabidopsis thaliana following thermal-stress. Plant Physiol. Biochem. 49, 235-243. doi: 10.1016/j. plaphy.2010.11.002

Filippou, P., Antoniou, C., Yelamanchili, S., and Fotopoulos, V. (2012). NO loading: efficiency assessment of five commonly used application methods of sodium nitroprusside in Medicago truncatula plants. Plant Physiol. Biochem. 60, 115-118. doi: 10.1016/j.plaphy.2012.07.026

Foyer, C. H., and Noctor, G. (2011). Ascorbate and glutathione: the heart of the redox hub. Plant Physiol. 155, 2-18. doi: 10.1104/pp.110.167569

Fu, J., and Huang, B. (2001). Involvement of antioxidants and lipid peroxidation in the adaptation of two cool-season grasses to localized drought stress. Environ. Exp. Bot. 45, 105-114. doi: 10.1016/S0098-8472(00)00084-8 the activities of the thermal phase of the photosystem, which contributed to limiting the energy input in the photosystem of tall fescue against heat stress. Synthesis of Asc increased under heat stress. However, this regulation did not occur at the transcription level and requires our further study.

\section{AUTHOR CONTRIBUTIONS}

$\mathrm{KC}, \mathrm{MZ}, \mathrm{HZ}$, and QZ wrote the main manuscript text; $\mathrm{MH}$ and DT prepared figures; XH and JL prepared the tables, JS and JF designed the overall of this study. All authors reviewed the manuscript.

\section{ACKNOWLEDGMENTS}

This work was financially supported by the National Natural Science Foundation of China (41503067, 21477165, 21503283, and C020406), the National Sci-Tech Support Plan (2015BAB01B04) and the Fundamental Research Funds for the Central Universities (CZQ15010).

Havaux, M. (1996). Short-term responses of photosystem I to heat stress Induction of a PS II-independent electron transport through PS I fed by stromal components. Photosynth. Res. 47, 85-97. doi: 10.1007/Bf00017756

Hu, T., Sun, X., Zhang, X., Nevo, E., and Fu, J. (2014). An RNA sequencing transcriptome analysis of the high-temperature stressed tall fescue reveals novel insights into plant thermotolerance. BMC Genomics 15:1147. doi: 10.1186/ 1471-2164-15-1147

Li, J., Liang, D., Li, M., and Ma, F. (2013). Light and abiotic stresses regulate the expression of GDP-L-galactose phosphorylase and levels of ascorbic acid in two kiwifruit genotypes via light-responsive and stress-inducible cis-elements in their promoters. Planta 238, 535-547. doi: 10.1007/s00425-013-1915-z

Linster, C. L., and Clarke, S. G. (2008). L-Ascorbate biosynthesis in higher plants: the role of VTC2. Trends Plant Sci. 13, 567-573. doi: 10.1016/j.tplants.2008. 08.005

Lu, M., and Zhang, Z.-Z. (2014). Phytoremediation of soil co-contaminated with heavy metals and deca-BDE by co-planting of Sedum alfredii with tall fescue associated with Bacillus cereus JP12. Plant Soil 382, 89-102. doi: 10.1007/ s11104-014-2147-0

Luo, H., Li, H., Zhang, X., and Fu, J. (2011). Antioxidant responses and gene expression in perennial ryegrass (Lolium perenne L.) under cadmium stress. Ecotoxicology 20, 770-778. doi: 10.1007/s10646-011-0628-y

Mehta, P., Allakhverdiev, S. I., and Jajoo, A. (2010). Characterization of photosystem II heterogeneity in response to high salt stress in wheat leaves (Triticum aestivum). Photosynth. Res. 105, 249-255. doi: 10.1007/s11120-0109588-y

Mellidou, I., Keulemans, J., Kanellis, A. K., and Davey, M. W. (2012). Regulation of fruit ascorbic acid concentrations during ripening in high and low vitamin $\mathrm{C}$ tomato cultivars. BMC Plant Biol. 12:239. doi: 10.1186/1471-2229-12-239

Mian, M. R., Zhang, Y., Wang, Z.-Y., Zhang, J.-Y., Cheng, X., Chen, L., et al. (2008). Analysis of tall fescue ESTs representing different abiotic stresses, tissue types and developmental stages. BMC Plant Biol. 8:27. doi: 10.1186/14712229-8-27

Nakano, Y., and Asada, K. (1981). Hydrogen peroxide is scavenged by ascorbatespecific peroxidase in spinach chloroplasts. Plant Cell Physiol. 22, 867-880. doi: 10.1093/oxfordjournals.pcp.a076232

Pfannschmidt, T. (2003). Chloroplast redox signals: how photosynthesis controls its own genes. Trends Plant Sci. 8, 33-41. doi: 10.1016/s1360-1385(02)00005-5

Pospísil, P. (2012). Molecular mechanisms of production and scavenging of reactive oxygen species by photosystem II. Biochim. Biophys. Acta 1817, 218-231. doi: 10.1016/j.bbabio.2011.05.017 
Ramel, F., Sulmon, C., Bogard, M., Couee, I., and Gouesbet, G. (2009). Differential patterns of reactive oxygen species and antioxidative mechanisms during atrazine injury and sucrose-induced tolerance in Arabidopsis thaliana plantlets. BMC Plant Biol. 9:28. doi: 10.1186/1471-2229-9-28

Robinson, H. H., and Yocum, C. F. (1980). Cyclic photophosphorylation reactions catalyzed by ferredoxin, methyl viologen and anthraquinone sulfonate. Use of photochemical reactions to optimize redox poising. Biochim. Biophys. Acta 590, 97-106. doi: 10.1016/0005-2728(80)90149-8

Smirnoff, N. (2011). Vitamin C: the metabolism and functions of ascorbic acid in plants. Adv. Bot. Res. 59, 107-177. doi: 10.1007/s10725-013-9845-0

Smirnoff, N., and Wheeler, G. L. (2000). Ascorbic acid in plants: biosynthesis and function. Crit. Rev. Plant Sci. 19, 267-290. doi: 10.1080/07352680091139231

Srivastava, A., Guisse, B., Greppin, H., and Strasser, R. J. (1997). Regulation of antenna structure and electron transport in photosystem II of Pisum sativum under elevated temperature probed by the fast polyphasic chlorophyll a fluorescence transient: OKJIP. Biochim. Biophys. Acta 1320, 95-106. doi: 10.1016/s0005-2728(97)00017-0

Stirbet, A. (2012). Chlorophyll a fluorescence induction: a personal perspective of the thermal phase, the J-I-P rise. Photosynth. Res. 113, 15-61. doi: 10.1007/ s11120-012-9754-5

Stirbet, A., and Govindjee (2011). On the relation between the Kautsky effect (chlorophyll a fluorescence induction) and Photosystem II: basics and applications of the OJIP fluorescence transient. J. Photochem. Photobiol. B 104, 236-257. doi: 10.1016/j.jphotobiol.2010.12.010

Sun, X. Y., Du, Z. M., Ren, J., Amombo, E., Hu, T., and Fu, J. M. (2015). Association of SSR markers with functional traits from heat stress in diverse tall fescue accessions. BMC Plant Biol. 15:116. doi: 10.1186/s12870-0150494-5

Takahashi, S., Nakamura, T., Sakamizu, M., van Woesik, R., and Yamasaki, H. (2004). Repair machinery of symbiotic photosynthesis as the primary target of heat stress for reef-building corals. Plant Cell Physiol. 45, 251-255. doi: $10.1093 / \mathrm{pcp} / \mathrm{pch} 028$

Tang, Y. L., Wen, X. G., Lu, Q. T., Yang, Z. P., Cheng, Z. K., and Lu, C. M. (2007). Heat stress induces an aggregation of the light-harvesting complex of photosystem II in spinach plants. Plant Physiol. 143, 629-638. doi: 10.1104/pp. 106.090712

Torabinejad, J., Donahue, J. L., Gunesekera, B. N., Allen-Daniels, M. J., and Gillaspy, G. E. (2009). VTC4 Is a bifunctional enzyme that affects myoinositol and ascorbate biosynthesis in plants. Plant Physiol. 150, 951-961. doi: 10.1104/ pp.108.135129
Toth, S. Z., Nagy, V., Puthur, J. T., Kovacs, L., and Garab, G. (2011). The physiological role of ascorbate as photosystem II electron donor: protection against photoinactivation in heat-stressed leaves. Plant Physiol. 156, 382-392. doi: 10.1104/pp.110.171918

Toth, S. Z., Puthur, J. T., Nagy, V., and Garab, G. (2009). Experimental evidence for ascorbate-dependent electron transport in leaves with inactive oxygen-evolving complexes. Plant Physiol. 149, 1568-1578. doi: 10.1104/pp.108.132621

Tsimilli-Michael, M., and Strasser, R. (2008). "Experimental resolution and theoretical complexity determine the amount of information extractable from the chlorophyll fluorescence transient OJIP," in Proceedings of the 14th International Congress on Photosynthesis: Photosynthesis Energy from the Sun, Vol. 1, eds J. Allen, E. Gantt, J. Golbeck, and B. Osmond (Dordrecht: Springer), 697-701.

Uchida, A., Jagendorf, A. T., Hibino, T., Takabe, T., and Takabe, T. (2002). Effects of hydrogen peroxide and nitric oxide on both salt and heat stress tolerance in rice. Plant Sci. 163, 515-523. doi: 10.1016/S0168-9452(02)00159-0

Umena, Y., Kawakami, K., Shen, J.-R., and Kamiya, N. (2011). Crystal structure of oxygen-evolving photosystem II at a resolution of 1.9 angstrom. Nature 473, 55-60. doi: 10.1038/nature09913

Wang, X. Y., and Huang, B. R. (2017). Lipid- and calcium-signaling regulation of HsfA2c-mediated heat tolerance in tall fescue. Environ. Exp. Bot. 136, 59-67. doi: 10.1016/j.envexpbot.2017.01.008

Wheeler, G. L., Jones, M. A., and Smirnoff, N. (1998). The biosynthetic pathway of vitamin C in higher plants. Nature 393, 365-369. doi: 10.1038/30728

Yamashita, A., Nijo, N., Pospisil, P., Morita, N., Takenaka, D., Aminaka, R., et al. (2008). Quality control of photosystem II - Reactive oxygen species are responsible for the damage to photosystem II under moderate heat stress. J. Biol. Chem. 283, 28380-28391. doi: 10.1074/Jbc.M710465200

Conflict of Interest Statement: The authors declare that the research was conducted in the absence of any commercial or financial relationships that could be construed as a potential conflict of interest.

Copyright (c) 2017 Chen, Zhang, Zhu, Huang, Zhu, Tang, Han, Li, Sun and Fu. This is an open-access article distributed under the terms of the Creative Commons Attribution License (CC BY). The use, distribution or reproduction in other forums is permitted, provided the original author(s) or licensor are credited and that the original publication in this journal is cited, in accordance with accepted academic practice. No use, distribution or reproduction is permitted which does not comply with these terms. 